\section{HPV-16 E2 gene disruption and sequence variation in CIN 3 lesions and invasive squamous cell carcinomas of the cervix: relation to numerical chromosome abnormalities}

\author{
D A Graham, C S Herrington
}

\begin{abstract}
Aim-To test the hypothesis that, because the human papillomavirus (HPV) E2 protein represses viral early gene transcription, E2 gene sequence variation or disruption could play a part in the induction of the numerical chromosome abnormalities that have been described in squamous cervical lesions.

Methods-The integrity and sequence of the E2 gene from 11 cervical intraepithelial neoplasia (CIN) grade 3 lesions and 14 invasive squamous cell carcinomas, all of which contained HPV-16, were analysed by the polymerase chain reaction (PCR). The E2 gene was amplified in three overlapping fragments and PCR products sequenced directly. Chromosome abnormalities were identified by interphase cytogenetics using chromosome specific probes for chromosomes $1,3,11,17,18$, and $X$.
\end{abstract}

Results-E2 gene disruption was present in significantly more invasive carcinomas (eight of 14) than CIN 3 lesions (one of 11) $(p=0.03)$. No association was found between E2 disruption and the presence of a numerical chromosome abnormality. The E2 gene from the non-disrupted isolates was sequenced and wild-type $(n=5)$ and variant $(n=11)$ sequences identified. Variant sequences belonged to European and African classes and contained from one to 15 amino acid substitutions. Although numerical chromosome abnormalities were significantly more frequent in invasive squamous cell carcinoma than CIN $3(p=0.04)$, there was no significant relation between the presence of sequence variation and either histological diagnosis or chromosome abnormality.

Conclusions-These data do not support the hypothesis that E2 gene disruption or variation is important in the induction of chromosome imbalance in these lesions. However, there is a relation between E2 gene disruption and the presence of invasive disease.

(F Clin Pathol: Mol Pathol 2000;53:201-206)

Keywords: papillomavirus; E2 gene; cervix; chromosome

Human papillomaviruses (HPV) are DNA tumour viruses that are associated with the formation of epithelial tumours. Over 80 types of HPV have now been described and are categorised as low risk (for example, HPV-6 and
HPV-11), intermediate risk (for example, HPV-31 and HPV-33), and high risk (for example, HPV-16 and HPV-18), based on their association with clinical disease. ${ }^{1}$ The HPV genome encodes two classes of genes that have been divided into two functional groups; the early (E) genes (E1, E2, E4, E5, E6, and E7) and the late (L) genes (L1 and L2). The E6 and E7 genes encode oncoproteins that, among other properties, bind to the p53 and retinoblastoma ( $\mathrm{pRb}$ ) proteins, respectively. The E1 protein is involved in viral DNA replication, the $\mathrm{E} 4$ protein in productive viral infection, and the E5 protein has some transforming properties. The L1 and L2 genes encode the major and minor capsid proteins, respectively.

The papillomavirus E2 protein is a modulator of papillomavirus transcription and replication, having major roles in viral DNA replication and as a repressor of viral early gene transcription. ${ }^{2-6}$ It has three functional domains: the $\mathrm{N}$-terminal transactivation domain, the hinge region, and the C-terminal DNA binding domain. ${ }^{7-9}$ In vitro studies have shown that the HPV-16 and HPV-18 early promoters, which regulate the transcription of the oncogenic E6 and E7 genes, are regulated by the viral E2 protein..$^{11}$ The expression of both the E6 and E7 genes of the high risk HPVs is necessary for the efficient immortalisation of primary human keratinocytes, ${ }^{12}{ }^{13}$ and the E2 protein is able to repress E6 and E7 expression by binding to sites adjacent to the major early promoter. ${ }^{14}$ The integration of HPV DNA into host cellular DNA is associated with neoplastic progression ${ }^{15}$ and, when HPV integrates, the viral DNA frequently breaks in the E1-E2 region. ${ }^{16}$ Consequently, disruption of the $\mathrm{E} 2$ open reading frame results in loss of $\mathrm{E} 2$ protein function, leading to uncontrolled E6 and E7 gene expression. This is also in keeping with the fact that E2 gene mutation or disruption can increase the immortalisation capacity of HPV-16. ${ }^{15} 17$

In a previous study, we identified distinct patterns of numerical chromosome abnormality in high grade intraepithelial and invasive squamous lesions of the cervix. ${ }^{18}$ In view of the in vitro evidence that the E2 protein can abrogate a mitotic control checkpoint, ${ }^{19}$ we hypothesised that the presence of numerical chromosome abnormalities in these lesions might be associated with the retention of intact E2 genes. Similarly, because mutation of the E2 gene can alter viral immortalisation capacity, ${ }^{15}$ we analysed the DNA sequence of the E2 gene in those lesions in which it was not disrupted to 
test the hypothesis that, in the absence of disruption, sequence variation might be important in the induction of chromosome abnormalities.

\section{Materials and methods}

CLINICAL MATERIAL

Eleven HPV-16 positive cervical intraepithelial neoplasia (CIN) grade 3 lesions and 14 HPV-16 positive invasive squamous cell carcinomas from our previous study of chromosome abnormalities ${ }^{18}$ were included in our present study. HPV-16 was identified using the GP5+/ GP6+ generic PCR system and dot blot hybridisation, as described previously. ${ }^{18} 20$

\section{ANALYSIS OF E2 GENE INTEGRITY}

DNA quality was demonstrated by amplification of a $536 \mathrm{bp} \beta$-globin fragment. ${ }^{21}$ The E2 gene was amplified in three overlapping fragments (amplimers A, B, and C), each of which is smaller in size than the internal control amplimer, using the following primer pairs. Nucleotide positions (nt) are according to the HPV-16R sequence. ${ }^{22}$

Amplimer A (475 bp product): A1, 5'-AGGA CGAGGACAAGGAAAA-3' (nt 2735-2753); A2, 5'-ACTTGACCCTCTACCACAGTTA CT-3' (nt 3187-3210). Amplimer B (477 bp product): B1 5'-TTGTGAAGAAGCATCAGT AACT-3' (nt 3172-3193); B2, 5'-TAAAGTAT TAGCATCACCTT-3' (nt 3630-3649). Amplimer C (276 bp product): C1, 5'-GTAATAG TAACACTACACCCATA-3' (nt 3597-3618); C2, 5'-GGATGCAGTATCAAGATTTGTT-3' (nt 3853-3873).

These primers were validated using DNA extracted from paraffin wax embedded CaSki and $\mathrm{SiHa}$ cells, which contain intact and disrupted E2 genes, respectively. ${ }^{23}{ }^{24}$ DNA was extracted from three $6 \mu \mathrm{m}$ thick, formalin

Table 1 Summary of the relation between E2 gene disruption, histological diagnosis, and interphase cytogenetic data

\begin{tabular}{|c|c|c|c|c|c|c|c|c|}
\hline \multirow[b]{2}{*}{ Diagnosis } & \multirow[b]{2}{*}{ Karyotype } & \multicolumn{6}{|c|}{ Chromosome number } & \multirow{2}{*}{$\begin{array}{l}\text { Non-amplifying } \\
\text { Segment }(s)\end{array}$} \\
\hline & & 1 & 3 & 11 & 17 & 18 & $X$ & \\
\hline CIN 3 & Aneusomic & 4 & 4 & 3 & 3 & 3 & 5 & None \\
\hline CIN 3 & Aneusomic & 2 & 2 & 2 & 2 & 3 & 4 & None \\
\hline CIN 3 & Tetrasomic & 4 & 4 & 4 & 4 & 4 & 4 & None \\
\hline CIN 3 & Disomic & 2 & 2 & 2 & 2 & 2 & 2 & None \\
\hline CIN 3 & Disomic & 2 & 2 & 2 & 2 & 2 & 2 & None \\
\hline CIN 3 & Disomic & 2 & 2 & 2 & 2 & 2 & 2 & None \\
\hline CIN 3 & Disomic & 2 & 2 & 2 & 2 & 2 & 2 & None \\
\hline CIN 3 & Disomic & 2 & 2 & 2 & 2 & 2 & 2 & None \\
\hline CIN 3 & Disomic & 2 & 2 & 2 & 2 & 2 & 2 & None \\
\hline CIN 3 & Disomic & 2 & 2 & 2 & 2 & 2 & 2 & None \\
\hline CIN 3 & Disomic & 2 & 2 & 2 & 2 & 2 & 2 & $\mathrm{~B}, \mathrm{C}$ \\
\hline ISCC & Aneusomic & 2 & 2 & 2 & 2 & 2 & 1 & None \\
\hline ISCC & Aneusomic & 3 & 3 & 3 & 3 & 3 & 5 & None \\
\hline ISCC & Tetrasomic & 4 & 4 & 4 & 4 & 4 & 4 & None \\
\hline ISCC & Aneusomic & 4 & 2 & 4 & 4 & 2 & 4 & None \\
\hline ISCC & Aneusomic & 4 & 3 & 1 & 3 & 4 & 4 & None \\
\hline ISCC & Aneusomic & 2 & 3 & 2 & 2 & 2 & 3 & None \\
\hline ISCC & Aneusomic & 3 & 4 & 2 & 2 & 2 & 4 & B \\
\hline ISCC & Aneusomic & 4 & 4 & 4 & 3 & 4 & 5 & $\mathrm{~B}$ \\
\hline ISCC & Disomic & 2 & 2 & 2 & 2 & 2 & 2 & $\mathrm{~A}, \mathrm{~B}, \mathrm{C}$ \\
\hline ISCC & Disomic & 2 & 2 & 2 & 2 & 2 & 2 & $\mathrm{~B}, \mathrm{C}$ \\
\hline ISCC & Aneusomic & 2 & 2 & 3 & 2 & 2 & 3 & $\mathrm{~A}$ \\
\hline ISCC & Aneusomic & 3 & 3 & 2 & 2 & 2 & 3 & A, B \\
\hline ISCC & Disomic & 2 & 2 & 2 & 2 & 2 & 2 & $\mathrm{~A}$ \\
\hline ISCC & Disomic & 2 & 2 & 2 & 2 & 2 & 2 & $\mathrm{~A}, \mathrm{~B}$ \\
\hline
\end{tabular}

The data for each chromosome refer to the number of chromosomes identified, as reported previously. ${ }^{18}$

The definitions of aneusomic, tetrasomic, and disomic are given in the text.

CIN 3, cervical intraepithelial neoplasia grade 3; ISCC, invasive squamous cell carcinoma. fixed, paraffin wax embedded sections using proteinase K (Boehringer-Mannheim, Lewes, Sussex, UK) and the supernatant treated with Instagene (Bio-Rad, Hemel Hempstead, Hertfordshire, UK) to remove cellular debris, as described previously. ${ }^{25}$ An aliquot of $20 \mu \mathrm{l}$ of DNA was used for each PCR reaction, which contained, in a total volume of $50 \mu 1,1 \times$ PCR buffer (Gibco BRL, Paisley, Scotland, UK), $1.5 \mathrm{mM} \mathrm{MgCl}, 0.2 \mathrm{mM}$ of each dNTP, and 1.25 units of AmpliTaq DNA polymerase. Hot start was achieved using Ampliwax PCR gems (Perkin-Elmer, Warrington, Cheshire, UK) and the following parameters were used: $95^{\circ} \mathrm{C}$ for four minutes then 40 cycles of $95^{\circ} \mathrm{C}$ for one minute, $58^{\circ} \mathrm{C}$ for two minutes, and $72^{\circ} \mathrm{C}$ for 1.5 minutes, followed by $72^{\circ} \mathrm{C}$ for seven minutes and a final resting temperature of $4^{\circ} \mathrm{C}$. The reference HPV-16 clone (obtained from Dr E-M deVilliers, Heidelberg, Germany) and DNA extracted from paraffin wax embedded CaSki cells were amplified as positive controls. The omission of template DNA served as a negative control. Detection was performed by running $10 \mu \mathrm{l}$ of amplified DNA on a $1.5 \%$ agarose gel, staining with ethidium bromide, and photographing over an ultraviolet transilluminator.

\section{E2 SEQUENCE ANALYSIS}

PCR products from the isolates, the HPV-16 clone, and CaSki cells were purified from agarose gels using the QIAquick ${ }^{\mathrm{TM}}$ gel extraction kit (Qiagen Ltd, Crawley, Sussex, UK) and then sequenced bidirectionally with the primer pair used to produce the amplicon. A further primer pair (5'-TACAAGACGTTAGCCTTG AAG-3' and 5'-ACCCGCATGAACTTCC CATAC-3') was used to amplify the region from 3036 to 3322 because the initial primer pairs produced poor quality sequence in this region. All sequencing was performed in an ABI Prism ${ }^{\mathrm{TM}} 373$ automated sequencer. Each sequenced region was then aligned with the wild-type HPV-16R E2 sequence ${ }^{22}$ to identify sequence variation. All sequences were confirmed bidirectionally.

CHROMOSOME ANALYSIS

Interphase cytogenetics was performed in each case for chromosomes 1, 3, 11, 17, 18, and X using pericentromeric probes, as described previously. ${ }^{1825}$

\section{STATISTICS}

Proportions were compared using Fisher's exact test and a significance level of $p<0.05$ was used.

\section{Results}

INTERPHASE CYTOGENETICS

The lesions that were used in our study had been investigated previously for numerical chromosome abnormalities using pericentromeric chromosome probes specific for chromosomes $1,3,11,17,18$, and $\mathrm{X} .{ }^{18}$ Lesions were classified as disomic (no numerical chromosome abnormality), tetrasomic (duplication of chromosome number with no imbalance), or aneusomic (numerical chromosome imbal- 

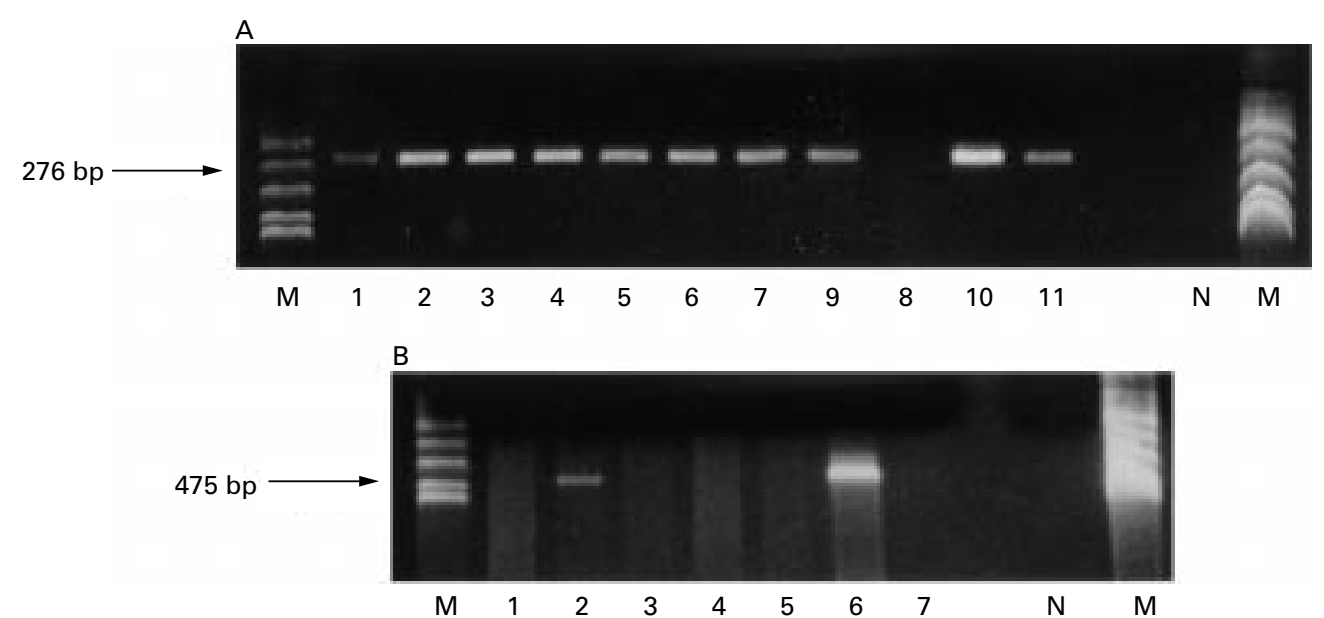

Figure 1 (A) Gel photograph of PCR performed on cervical intraepithelial neoplasia grade 3 (CIN 3) lesions with primers $C 1$ and C2, demonstrating failure of amplification of this region of the E2 gene in one of 11 lesions. (B) Gel photograph of PCR performed on invasive squamous carcinomas with primers $A 1$ and A2, demonstrating failure of amplification of this region of the E2 gene in five of seven lesions. $N$, water blank negative control; M, marker (Msp I digested $p B R 322)$.

ance) (table 1). Aneusomy was present in significantly more invasive carcinomas (nine of 14) than CIN 3 lesions (two of 11) ( $p=0.04)$.

DISRUPTION OF THE E2 GENE

The overlapping primer pairs were validated initially using DNA extracted from CaSki and SiHa cells: all three fragments were amplifiable from CaSki cells but only one (amplimer C) could be amplified from $\mathrm{SiHa}$ cells, as predicted from the published sequences. ${ }^{24}$ The $536 \mathrm{bp} \beta$-globin fragment was amplified in all 25 cases. Amplification of the HPV-16 E2 gene from these lesions demonstrated a failure of amplification of one or more amplimer, indicating disruption, in significantly more invasive squamous cell carcinomas (eight of 14) than CIN 3 lesions (one of 11) ( $p=0.03$; fig 1). There was, however, no significant relation between the presence of aneusomy and E2 gene disruption $(\mathrm{p}=1$; table 1$)$.

E2 GENE SEQUENCE ANALYSIS

The HPV-16 isolates that did not show evidence of disruption were sequenced, as were amplimers from the HPV-16 clone and from CaSki cells, and compared with the wild-type HPV-16R E2 reference sequence ${ }^{22}$ to investigate variation within the E2 gene. Mutations were identified in a total of 24 positions, 18 of which resulted in amino acid changes (fig 2). A guanosine residue was also identified in position 2926 in all isolates, in addition to CaSki cells and the HPV-16 clone: this has been identified recently as a sequencing error in the HPV-16R sequence. ${ }^{22}$ Several consistent variants were identified. The most common individual base change was $\mathrm{C}$ to $\mathrm{T}$ at position 2410. This was present in isolates in which it was the only variant residue, or in which it was associated with other single base changes (group 1). It was also present in variants that showed more widespread variation (groups 2 and 3). Group 2 variants had the same sequence as the E2 gene in CaSki cells. ${ }^{24}$ Group 3 variants showed a much greater variation, with a total of 20 variant positions. Overall, there was no relation between the presence of E2 mutation and either histological diagnosis $(p=1.0)$ or chromosome imbalance $(p=1.0)$.

Variation in the transactivation domain was only identified in groups 2 and 3 . In group 2, the single mutation in this domain is silent. In group 3, there are five mutations that lead to amino acid substitutions. Variation was also observed in the hinge and DNA binding regions, with a greater number of mutations again being present in the group 3 variants. Two different mutations were identified at position 3377: $\mathrm{C}$ to $\mathrm{G}$ was present in group 3, and produces a proline to arginine amino acid substitution; $\mathrm{C}$ to $\mathrm{T}$, which produces a proline to serine substitution, was identified in a single isolate in group 1. Some mutations within the hinge region also affect the sequence of the E4 gene. These were identified only in group 3 variants and are as follows: L62T (3516, C to A and 3517, $\mathrm{T}$ to $\mathrm{C}$ ); Q69P (3538, $\mathrm{A}$ to $\mathrm{C}$ ); H78Q (3566, T to G).

\section{Discussion}

Disruption of the E2 gene was significantly more frequent in invasive squamous cell carcinomas than in CIN 3 lesions. Although this is in keeping with viral integration being important in neoplastic progression, disruption of the E2 gene does not necessarily equate to viral integration. E2 genes can be intact in the presence of viral integration-for example, when both episomal and integrated sequences are present within a lesion, or in the presence of integration of multiple copies of the viral genome, as occurs in CaSki cells. ${ }^{23}$ No relation was found between E2 gene disruption and the presence of numerical chromosome abnormalities, as defined using pericentromeric repeat probes. More specifically, chromosome imbalance (aneusomy) was identified both in lesions with disrupted E2 genes and in those with intact E2 genes. These data do not support the hypothesis that the presence of an intact E2 gene is important in the induction of numerical chromosome abnormalities. 


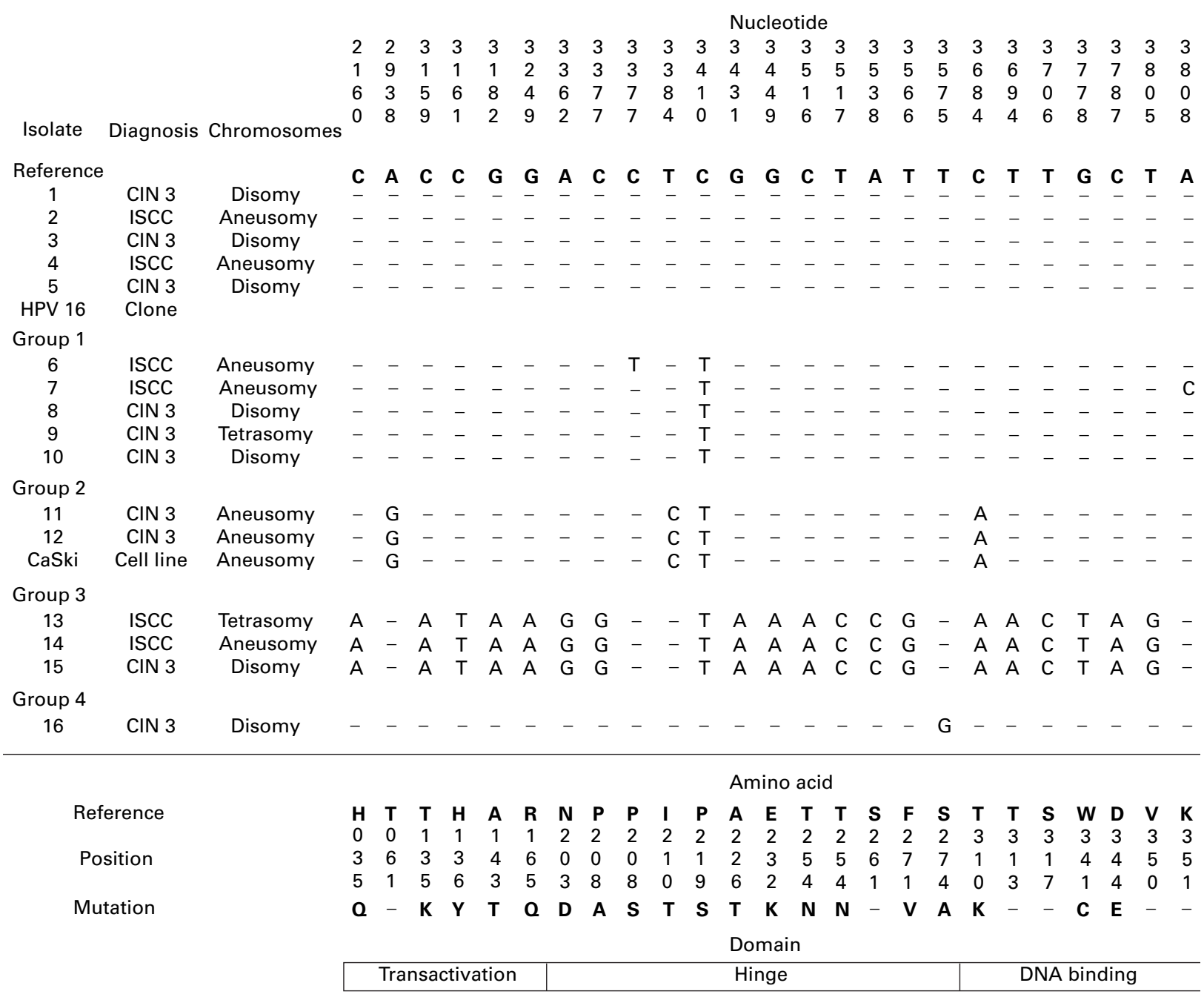

Figure 2 Summary of the position of the E2 mutations identified in the human papillomavirus 16 (HPV-16) isolates. The isolates are grouped according to the combination of mutations identified. The resulting amino acid substitution (if any) is given below each variant position. GenBank accession numbers have been assigned as follows: AF193425 (isolate 6); AF193426 (isolate 7); AF193427 (isolates 8-10); AF193428 (isolates 11 and 12); AF193429 (isolates 13-15); AF193430 (isolate 16). CIN 3, cervical intraepithelial neoplasia grade 3; ISCC, invasive squamous cell carcinoma.

Within HPV-16, five major phylogenetic branches have been defined on the basis of variation within the E6 and L1 genes, each predominating within specific geographical regions: these branches have been designated $\mathrm{E}$ (European), As (Asian), AA (AsianAmerican), and Af (African) ${ }^{26} \mathrm{~A}$ recent report that details variation in the $\mathrm{E} 2$ hinge region and its relation to the more extensively studied E6 and $\mathrm{L} 1$ variants allows the approximate identification of the groups of isolates identified in our study. ${ }^{27}$ Group 1 variants show the $3410 \mathrm{C}$ to $\mathrm{T}$ mutation alone or in combination with unique mutations elsewhere. This mutation is found in $\mathrm{E}, \mathrm{AA}$, and Af variants but, when it is the only mutation in the hinge region, it is restricted to $\mathrm{E}$ variants. Group 2 variants also belong to the $\mathrm{E}$ cluster. Moreover, the sequence of the transactivation and DNA binding domains is identical to the sequence of the HPV-16 E2 gene present in CaSki cells, both in our study and in data reported by others. ${ }^{24}$ The group 3 variants show mutations within the hinge region that are common to both AA and Af variants, but a comparison of the sequence with those reported by Eriksson $e t$ al identifies them as Af group 2a (Af2a) variants. ${ }^{27} \mathrm{~A}$ recent report detailing a series of AA variants ${ }^{28}$ supports this interpretation. Specifically, our group 3 isolates possess a $3431 \mathrm{G}$ to A mutation, which does not occur in AA variants, ${ }^{27}{ }^{28}$ and do not possess the $3224 \mathrm{~T}$ to A mutation reported in AAa and AAc variants, the $3181 \mathrm{~A}$ to $\mathrm{C}$ or $3387 \mathrm{~T}$ to $\mathrm{C}$ mutations reported in AAa variants, or the $3416 \mathrm{G}$ to $\mathrm{A}$ mutation identified in AAc variants. ${ }^{28}$ It is of note that Eriksson and colleagues ${ }^{27}$ did not find the 3362 A to $G$ mutation in AA variants, whereas Casas et al did. ${ }^{28}$ This does not affect the identification of our variants as Af2a.

Mutations in the transactivation domain were present both in group 2 and 3 variants. The single mutation in group 2 variants is silent and hence no amino acid substitutions in this domain are present in this group. By contrast, the group 3 variants contain several mutations that lead to amino acid substitutions in this domain. The H35Q mutation is within the E1 interaction site, suggesting that this change might affect the formation of E1-E2 heterodimers, which are known to be important in viral DNA replication. However, AA 
variants containing this mutation were shown to be capable of supporting viral DNA replication in carcinomas in which the E1-E2 region was retained, ${ }^{28}$ indicating that this mutation does not abrogate this function. Mutations within the hinge region are of less clear functional importance, although they could potentially alter the three dimensional relation between transactivation and DNA binding domains. This possibility is of particular relevance to the P219S (3410 C to $\mathrm{T}$ ) mutation, which occurred in all but one of the variant isolates, because the replacement of proline with serine at this position could significantly alter the secondary and tertiary protein structures. The frequency of this mutation raises the possibility that variants with this alteration could be involved in the development of high grade intraepithelial and invasive disease. However, an analysis of low grade intraepithelial lesions is required to assess this hypothesis.

The ability to bind to DNA, particularly that in the long control region of the HPV genome, is central to the function of the E2 protein. When binding to DNA, the E2 protein forms a homodimer, which interacts with the DNA molecule through its DNA binding helices formed from residues 292-309. Therefore, the T310K mutation, present both in group 2 and group 3 variants, might affect the three dimensional structure of this helix, and hence its ability to bind to DNA. However, a recent study showed very similar transcriptional activities when $\mathrm{E}$ and $\mathrm{AA}$ variants were compared. Although in vivo function cannot necessarily be inferred from in vitro functional studies, these data suggest that the amino acid sequence variation identified might not be relevant to this function of the protein.

Another possible effect of amino acid substitution in the E2 gene is an alteration of the host immune response to this protein. Linear epitopes have been described within the E2 protein: one in the transactivation domain (residues 121-140), three in the hinge region (residues 181-200, 241-260, and 271-290), and one in the DNA binding domain (residues 328-346). ${ }^{29-31}$ It is interesting that only the amino acid substitutions present in the group 3 variants, and that in the single group 4 isolate, affect these regions. Whether this is of biological relevance remains to be determined.

The lack of a relation between the presence of sequence variation and either histological diagnosis or chromosome imbalance suggests that any functional difference between the E2 variants is unlikely to be involved in determining either the development of aneusomy or progression to invasive carcinoma. However, these data do not exclude the possibility that the variants might differ in their ability to induce progression of a productive HPV-16 infection to CIN 3.

In conclusion, E2 gene disruption appears to be related to stromal invasion, but not to the presence of numerical abnormalities of chromosomes 1, 3, 11, 17, 18, or X. A variety of E2 gene variants were identified in the lesions analysed, but there was no association between the presence of these variations and either stromal invasion or chromosome abnormality. However, this does not exclude the possibility that E2 gene variation is important in determining the natural history of HPV-16 infection or the likelihood of progression to CIN 3. Further data are required regarding the prevalence of the $\mathrm{E} 2$ variants described across the spectrum of cervical squamous neoplasia.

We thank Wellbeing and the Royal College of Obstetricians and Gynaecologists, UK for funding the initial phase of this study.

1 Arends MJ, Buckley CH, Wells M. Aetiology, pathogenesis, and pathology of cervical neoplasia. $\mathcal{f}$ Clin Pathol 1998;51:96-103.

2 Spalholz BA, Yang YC, Howley PM. Transactivation of a bovine papillomavirus transcriptional regulatory element by the E2 gene product. Cell 1985;42:183-91.

3 Phelps WC, Howley PM. Transcriptional trans-activation by the human papillomavirus type $16 \mathrm{E} 2$ gene product. $f$ Virol 1987;61:1630-8.

4 Chiang C-M, Ustav M, Stenlund A, et al. Viral E1 and E2 proteins support replication of homologous and heteroloproteins support replication of homologous and heterolo89:5799-803.

5 Storey A, Piccini A, Massimi P, et al. Mutations in the human papillomavirus type $16 \mathrm{E} 2$ protein identify a region of the protein involved in binding to E1 protein. $\mathcal{F}$ Gen Virol 1995;76:819-26.

6 Zou N, Liu JS, Kuo SR, et al. The carboxyl-terminal region of the human papillomavirus type $16 \mathrm{E} 1$ protein determines E2 protein specificity during DNA replication. $f$ Virol 1998;72:3436-41.

7 Sanders CM, Stern PL, Maitland NJ. Characterization of human papillomavirus type $16 \mathrm{E} 2$ protein and subdomains expressed in insect cells. Virology 1995;211:418-33.

8 Hedge RS, Androphy EJ. Crystal structure of the E2 DNAbinding domain from human papillomavirus type 16: implications for its DNA binding-site selection mechanism. f Mol Biol 1998;284:1479-89.

9 Cooper CS, Upmeyer SN, Winokur PL. Identification of single amino acids in the human papillomavirus $11 \mathrm{E} 2$ prosingle amino acids in the human papillomavirus $11 \mathrm{E} 2$ pro-
tein critical for the transactivation or replication functions. Virology 1998;241:312-22.

10 Tan SH, Leong LE, Walker PA, et al. The human papillomavirus type $16 \mathrm{E} 2$ transcription factor binds with low cooperativity to two flanking sites and represses the E6 promoter through displacement of Sp1 and TFIID. $\mathcal{F}$ Virol 1994;68:6411-20.

11 Sanders CM, Maitland NJ. Kinetic and equilibrium binding studies of the human papillomavirus type-16 transcription regulatory protein E2 interacting with core enhancer elements. Nucleic Acids Res 1994;22:4890-7.

12 Sedman SA, Barbosa MS, Vass WC, et al. The full length E6 protein of human papillomavirus type 16 has transforming and transactivating activities and cooperates with E7 to immortalise keratinocytes in culture. F Virol 1991;65:48606.

13 Jewers RJ, Hildebrandt P, Ludlow JW, et al. Regions of human papillomavirus type $16 \mathrm{E} 7$ oncoprotein required for mmortalization of human keratinocytes. F Virol 1992;66: 1329-35

14 Dowhanick JJ, McBride AA, Howley PM. Suppression of cellular proliferation by the papillomavirus E2 protein. $\mathcal{F}$ Virol 1995;69:7791-9.

15 Romanczuk H, Howley PM. Disruption of either the E1 or the E2 regulatory gene of human papillomavirus type 16 increases viral immortalization capacity. Proc Natl Acad Sci US A 1992;89:3159-63.

16 Kalantari M, Karlsen F, Kristensen G, et al. Disruption of the E1 and E2 reading frames of HPV 16 in cervical carci1998; 17:146-53.

17 Storey A, Greenfield I, Banks L, et al. Lack of immortalizing activity of a human papillomavirus type 16 variant DNA with a mutation in the E2 gene isolated from normal human cervical keratinocytes. Oncogene 1992;7:459-65.

18 Graham DA, Southern SA, McDicken IW, et al. Interphase cytogenetic evidence for distinct genetic pathways in the development of squamous neoplasia of the uterine cervix. Lab Invest 1998;78:289-96.

19 Frattini MG, Hurst SD, Lim HB, et al. Abrogation of a mitotic checkpoint by E2 proteins from oncogenic human papillomaviruses correlates with increased turnover of the p53 tumor suppressor gene. EMBO F 1997;16:318-31.

20 Jacobs MV, Snijders PJF, van den Brule AJC, et al. A general primer GP5+/GP6+mediated PCR enzyme immunoassay method for rapid detection of 14 high-risk and 6 low-risk human papillomavirus genotypes in cervical scrapings. $\mathcal{f}$ Clin Microbiol 1997;35:791-5.

21 Bauer HM, Greer CE, Manos MM. Determination of genital human papillomavirus infection by consensus PCR amplification. In: Herrington, CS, McGee JO'D, eds. Diagamplification. In: Herrington, CS, McGee JO'D, eds. Diag-
nostic molecular pathology: a practical approach, Vol. II.
Oxford: Oxford University Press, 1992:131-52. Oxford: Oxford University Press, 1992:131-52. 
22 Meissner JD. Sequencing errors in reference HPV clones. In: Myers, G, Baker C, MungerK, et al, eds. Human papillomaviruses 1997: a compilation and analysis of nucleic acid and amino acid sequences. Los Alamos, New Mexico: Theoretical Biology and Biop

23 Baker CC, Phelps WC, Lindgren V, et al. Structural and transcriptional analysis of human papillomavirus type 16 sequences in cervical carcinoma cell lines. F Virol 1987;61 962-71.

24 Meissner JD. Nucleotide sequences and further characterization of human papillomavirus DNA present in CaSki, $\mathrm{SiHa}$ and HeLa cervical carcinoma cell lines. 7 Gen Virol 1999;80:1725-33.

25 Southern SA, Evans MF, Herrington CS. Basal cell tetrasomy occurs in low grade cervical squamous intraepithelial lesions infected with high risk human papillomaviruses. Cancer Res 1997;57:4210-13.

26 Yamada T, Manos MM, Peto J, et al. Human papillomavirus type 16 sequence variation in cervical cancers: a worldwide perspective. F Virol 1997;71:2463-72.
27 Eriksson A, Herron JR, Yamada T, et al. Human papillomavirus type 16 variant lineages characterised by nucleotide
sequence analysis of the E5 coding segment and the E2 hinge region. f Gen Virol 1999;80:595-600.

28 Casas L, Galvan SC, Ordonez RM, et al. Asian-American variants of human papillomavirus type 16 have extensive mutations in the E2 gene and are highly amplified in cervical carcinomas. Int f Cancer 1999;83:449-55.

29 Dillner J, Dillner L, Robb J, et al. A synthetic peptide defines a serological IgA response to a human papillomavirusencoded nuclear antigen expressed in virus carrying cervical neoplasia. Proc Natl Acad Sci U S A 1989;86:383841.

30 Dillner J, Wiklund F, Lenner P, et al. Antibodies against linear and conformational epitopes of human papillomavirus type 16 that independently associate with incident cervical cancer. Int f Cancer 1995;60:377-82.

31 Konya J, Eklund C, Geijersstam V, et al. Identification of a cytotoxic T-lymphocyte epitope in the human papillomavirus type 16 E2 protein. $\mathcal{F}$ Gen Virol 1997;78:2615-20. 